Original Article

\title{
Ezrin-radixin-moesin proteins are regulated by Akt-GSK3 $\beta$ signaling in the rat nucleus accumbens core
}

\author{
Wha Young Kim ${ }^{1, \#}$, Wen Ting Cai ${ }^{2, \#}$, Ju Kyong Jang ${ }^{3}$, and Jeong-Hoon Kim ${ }^{1,2, *}$ \\ Departments of ${ }^{1}$ Physiology and ${ }^{2}$ Medical Science, Brain Korea 21 Plus Project for Medical Science, Yonsei University College of Medicine, Seoul 03722 , \\ ${ }^{3}$ Bio-Pharm Solutions Co., Ltd., Suwon 16229, Korea
}

\section{ARTICLE INFO}

Received November 5, 2019

Revised November 15, 2019

Accepted November 20, 2019

*Correspondence

Jeong-Hoon Kim

E-mail:jkim1@yuhs.ac

\section{Key Words}

Drug addiction

Glycogen synthase kinase

Nucleus accumbens

Protein kinase B

Signal transduction

\#These authors contributed equally to this work.
ABSTRACT The ezrin-radixin-moesin (ERM) proteins are a family of membrane-associated proteins known to play roles in cell-shape determination as well as in signaling pathways. We have previously shown that amphetamine decreases phosphorylation levels of these proteins in the nucleus accumbens (NAcc), an important neuronal substrate mediating rewarding effects of drugs of abuse. In the present study, we further examined what molecular pathways may be involved in this process. By direct microinjection of LY294002, a PI3 kinase inhibitor, or of S9 peptide, a proposed GSK3 $\beta$ activator, into the NAcc core, we found that phosphorylation levels of ERM as well as of GSK3 $\beta$ in this site are simultaneously decreased. These results indicate that ERM proteins are under the regulation of Akt-GSK3 $\beta$ signaling pathway in the NAcc core. The present findings have a significant implication to a novel signal pathway possibly leading to structural plasticity in relation with drug addiction.

\section{INTRODUCTION}

Ezrin, radixin, and moesin proteins, collectively called as ERM, are a group of proteins with high degree of homology among themselves [1], and play important roles in cell-shape determination and structural stability by linking actin cytoskeleton to plasma membrane $[2,3]$. When phosphorylated at a threonine residue at $\mathrm{C}$-terminal regulatory domain, they become activated and bring F-actin close to plasma membrane, where they act as important mediators of cellular signaling pathways between outer and inner cellular compartments [1,4]. Thus, ERM proteins reside in a position that contribute to structural plasticity and changes of molecular signaling pathways linked to cytoskeletons $[3,5,6]$.

The nucleus accumbens (NAcc) is the brain site where drugs of abuse produce their rewarding effects [7-9]. The molecular changes in signaling pathways in this site are known to be critical for the expression of addictive behaviors [10]. Previously, we have found that ERM phosphorylation in this site is dose-dependently reduced by acute injection of cocaine [11], and especially in the core, a sub-region of the NAcc, by amphetamine (AMPH) [12]. Considering that ERM proteins are critical for the cellular structure, these findings suggest that ERM proteins may play a role in structural remodeling by taking a part in a series of psychomotor stimulant-induced cellular events in the NAcc.

While there is no information at all about how ERM proteins are regulated in the NAcc, we have previously shown that AMPH-induced decrease of ERM phosphorylation in the NAcc core, but not in the shell, are reversed by lithium [12]. Interestingly, it is shown that lithium activates protein kinase B (Akt) and subsequently inhibits glycogen synthase kinase $3 \beta$ (GSK3 $\beta$ ) by increasing their phosphorylation [13-15]. Put together, it is plausible to make a hypothesis that a signaling pathway mediated by
(1) \$ This is an Open Access article distributed under the terms of the Creative Commons Attribution Non-Commercial License, which permits unrestricted non-commercial use, distribution, and reproduction in any medium, provided the original work is properly cited. Copyright @ Korean J Physiol Pharmacol, pISSN 1226-4512, eISSN 2093-3827
Author contributions: W.Y.K., W.T.C. and J.-H.K. designed the experimental strategy and analyzed the data. W.Y.K., W.T.C. and J.K.J. performed all the experiments. J.-H.K. wrote the manuscript. All authors commented on the manuscript. 
Akt-GSK3 $\beta$ may also regulate ERM phosphorylation in the NAcc core. Supporting this possibility, it has been shown, although in neuronal and epidermal cell cultures, that ERM phosphorylation is under the regulation of Akt and its upstream effector, phosphatidylinositol 3-kinase (PI3 kinase) [16,17]. More recently, it has been also shown in murine auditory epithelial progenitor cells that Akt activation increases ERM phosphorylation levels [18]. Thus, we examined in the present study whether direct manipulation of Akt-GSK3 $\beta$ signaling in the NAcc core may influence ERM phosphorylation in this site.

\section{METHODS}

\section{Subjects}

Male Sprague-Dawley rats weighing 220-250 g (equivalent to 6 weeks olds) on arrival were obtained from Orient Bio Inc. (Seongnam-si, Korea). They were housed 3 per cage in a $12 \mathrm{~h}$ light/dark cycle room (lights out at 8:00 PM), and all experiments were conducted during the day time. The rats had access to food and water ad libitum at all times. All animal use procedures were conducted according to an approved Institutional Animal Care and Use Committee protocol (approval number: 09-140).

\section{Drugs and peptide}

LY294002 hydrochloride (Sigma-Aldrich, St. Louis, MO, USA), PI3 kinase inhibitor, was dissolved in 100\% DMSO and small aliquots were stored at $-80^{\circ} \mathrm{C}$. Immediately before use, frozen aliquots were diluted to final working concentrations of 0.8 or 8.0 $\mu \mathrm{g} / \mu \mathrm{l}$ in $80 \%$ DMSO. S9 peptide, which consists of 21 amino acids (a.a.) including a small peptide (11 a.a.), commonly referred to as protein transduction domain [19], and a portion (10 a.a.) of the $\mathrm{N}$-terminus sequence of GSK3 $\beta$ (GRPRTTSFAE) known as the substrate site for Akt and thereby competes with GSK3 $\beta$ against its phosphorylation [20,21], was artificially synthesized and kindly provided by Professor Soo Young Lee at the Center for Cell Signaling and Drug Discovery Research, Ewha Womans University (Seoul, Korea). It was dissolved to final working concentrations of 1.0 or $10.0 \mu \mathrm{g} / \mu \mathrm{l}$ in saline.
A

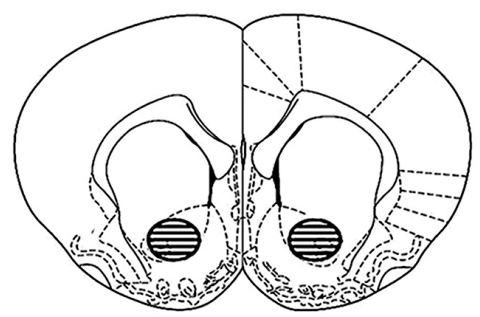

C

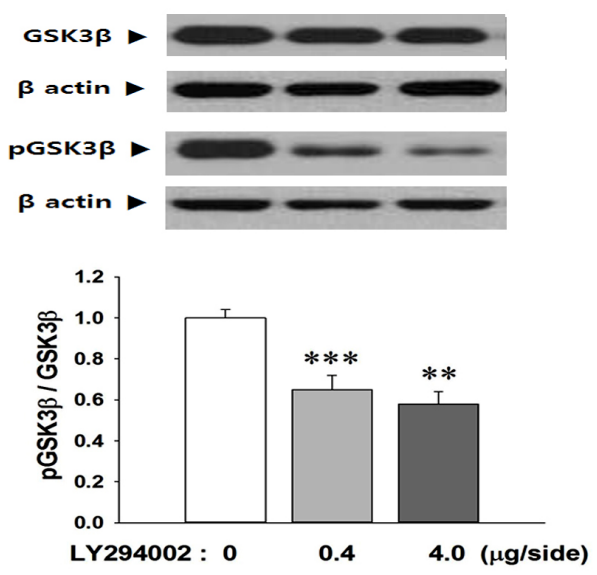

B

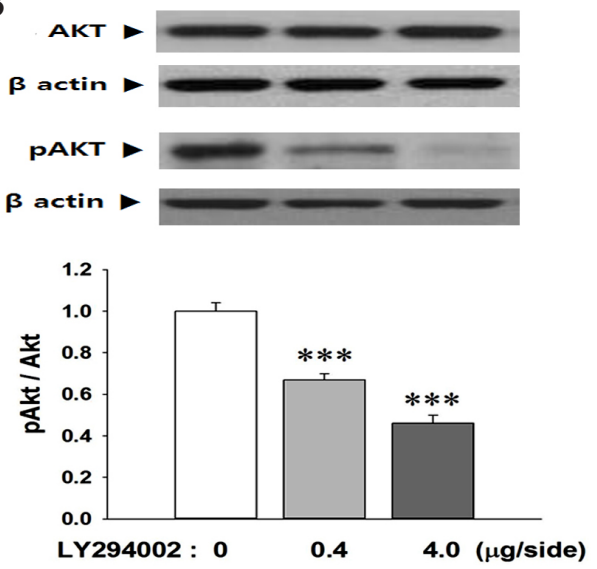

D
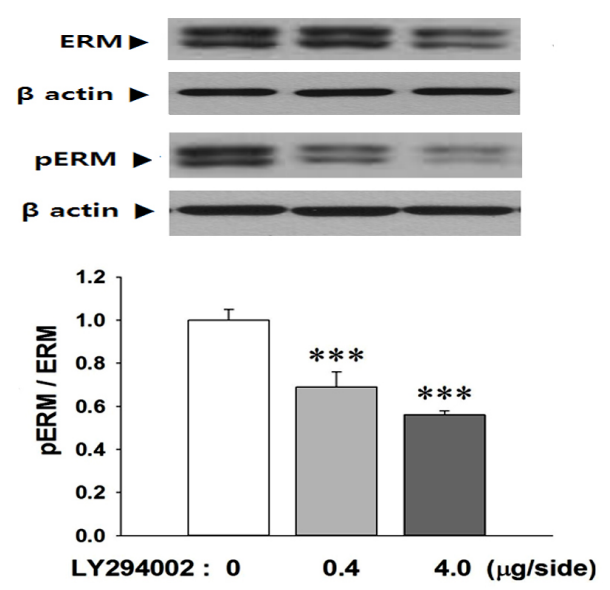

Fig. 1. Microinjection of LY294002 into the nucleus accumbens (NACC) core decreases phosphorylation levels of activates protein kinase B (Akt), glycogen synthase kinase $3 \beta$ (GSK3 $\beta$ ), and ezrin-radixin-moesin (ERM) in this site. (A) The NAcc core region where tissues were taken out is shown (cross-hatched circles). Punches (1.2 mm diameter) were prepared bilaterally and pooled for each individual animal's protein isolation. Line drawing is from Paxinos and Watson (2004) [32] and depicts the caudal surface of a coronal section ( $1.0 \mathrm{~mm}$ thick) extending 1.70-2.70 $\mathrm{mm}$ from bregma. (B-D) Representative Western blots are shown. Values for the band intensities were first normalized to $\beta$-actin and then the average values for the ratio of phosphorylated to total proteins in each group were expressed as mean + standard error of the mean relative to saline control group. Symbols indicate significant differences as revealed by post-hoc Bonferroni comparisons following oneway ANOVA. ${ }^{* *} p<0.01,{ }^{* * *} p<0.001$, significantly different compared to saline control rats. Numbers for each group are 6.

https://doi.org/10.4196/kjpp.2020.24.1.121 


\section{Surgical procedures}

Rats were anesthetized with intraperitoneal ketamine (100 mg/ $\mathrm{kg}$ ) and xylazine $(6 \mathrm{mg} / \mathrm{kg})$, placed in a stereotaxic instrument with the incisor bar at $5.0 \mathrm{~mm}$ above the interaural line and implanted with chronic bilateral guide cannulas (22 gauge tubing size with $0.71 \mathrm{~mm}$ in outer and $0.39 \mathrm{~mm}$ in internal diameters; Plastics One, Roanoke, VA, USA) aimed at the NAcc core (A/P, +3.4; L, \pm 1.5 ; D/V, $-7.5 \mathrm{~mm}$ from bregma and skull) [22]. Cannulas were angled at $10^{\circ}$ to the vertical, positioned $1 \mathrm{~mm}$ above the final injection site, and secured with dental acrylic cement anchored to stainless steel screws fixed to the skull. After surgery, 28 gauge (tubing size with $0.36 \mathrm{~mm}$ in outer and $0.18 \mathrm{~mm}$ in internal diameters) obturators were placed in the guide cannulas, and rats were returned to their home cages for a 7-day recovery period.

\section{Design and procedures}

Upon arrival, all rats passed a week-long adaptation period to the new housing environment. Then, bilateral guide cannula surgery was followed. About 1 week after surgery, three different groups of rats were bilaterally microinjected into the NAcc core with vehicle (80\% DMSO) or LY294002 (0.4 or $4.0 \mu \mathrm{g} / 0.5 \mu \mathrm{l} /$ side) (experiment 1), while a separate set of three different groups of rats were bilaterally microinjected into the NAcc core with vehicle (saline) or S9 peptide $(0.5$ or $5.0 \mu \mathrm{g} / 0.5 \mu \mathrm{l} /$ side) (experiment 2$)$. Bilateral intracranial microinjections into the NAcc core were made in the freely moving rat. Injection cannulas (28 gauge) connected to $1 \mu \mathrm{l}$ syringes (Hamilton, Reno, NV, USA) via PE-20 tubing were inserted to a depth $1 \mathrm{~mm}$ below the guide cannula tips. Injections were made in a volume of $0.5 \mu \mathrm{l}$ per side over 30 sec. After $1 \mathrm{~min}$, the injection cannulas were withdrawn and the obturators were replaced. Then, they were placed back to cage and let them rest for $60 \mathrm{~min}$, decapitated and the brain tissues (core only) were prepared for western blot analysis.

\section{Brain tissue preparation}

Animals were decapitated by guillotine $60 \mathrm{~min}$ after vehicle, drug, or peptide microinjections. Brains were rapidly removed and coronal sections (1.0 mm thick extending 1.60-2.60 mm from bregma) were obtained with an ice-cold brain slicer (Model SA-2160, Roboz Surgical Instrument Co., Gaithersburg, MD, USA). The NAcc core tissues were obtained in the circular punch

A

GSK3 $\beta$ (47 KD)
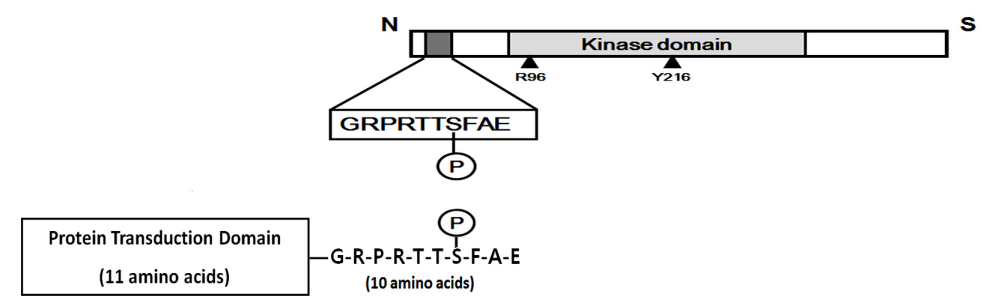

S9 peptide (21 amino acids)

B

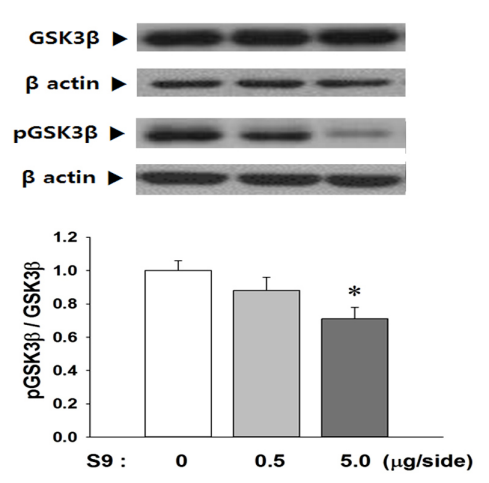

C

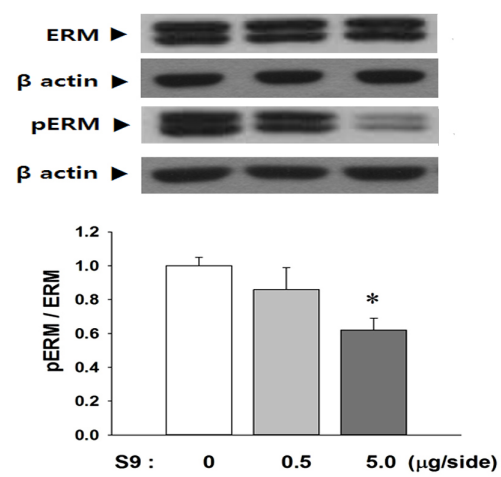

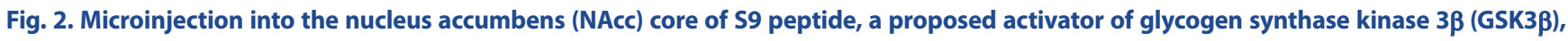
lowers phosphorylation levels of GSK3 $\beta$, and ezrin-radixin-moesin (ERM) in this site. (A) A diagram showing the S9 peptide with short amino acid sequences competitive against a phosphorylation site at serine 9 of endogenous GSK3 $\beta$. (B, C) Representative Western blots are shown. Values for the band intensities were first normalized to $\beta$-actin and then the average values for the ratio of phosphorylated to total proteins in each group were expressed as mean + standard error of the mean relative to saline control group. Symbols indicate significant differences as revealed by posthoc Bonferroni comparisons following one-way ANOVA. ${ }^{*} p<0.05$, significantly different compared to saline control rats. Numbers for each group are 5 to 6. 
with $1.2 \mathrm{~mm}$ diameter on an ice-cold plate (Fig. 1A), immediately frozen on dry ice and stored at $-80^{\circ} \mathrm{C}$. They were prepared bilaterally and pooled for each individual animal's protein isolation.

\section{Western blotting}

Tissues were homogenized in lysis buffer containing $0.32 \mathrm{M}$ sucrose, $2 \mathrm{mM}$ EDTA, $1 \% \mathrm{SDS}, 10 \mu \mathrm{g} / \mathrm{ml}$ aprotinin, $10 \mu \mathrm{g} / \mathrm{ml}$ leupeptin, $1 \mathrm{mM}$ phenylmethylsulfonyl fluoride, $10 \mathrm{mM}$ sodium fluoride, and $1 \mathrm{mM}$ sodium orthovanadate. The concentration of protein was determined by using Pierce Coomassie Protein Assay Kit (Thermo Scientific Inc., Rockford, IL, USA). Samples were then boiled for $10 \mathrm{~min}$ and subjected to SDS-polyacrylamide gel electrophoresis. Proteins were separated and transferred electrophoretically to nitrocellulose membranes (Bio-Rad, Hercules, CA, USA), which were then blocked with $5 \%$ bovine serum albumin (BSA) in PBS-T buffer (10 mM phosphate-buffered saline plus $0.05 \%$ Tween-20). Antibodies used to probe the blots were as following: total Akt (1:10,000), phosphor-Akt (1:500), total GSK3 $\beta$ (1:4,000), phosphor-GSK3 $\beta$ (specific to detect phosphorylated GSK3 $\beta$ at serine 9, 1:2,000), total ERM (1:1,000), phosphor-ERM (specific to detect phosphorylated ezrin-radixin-moesin at threonine 567, 564 or 558, respectively; 1:500), purchased from Cell Signaling (Beverly, MA, USA) and diluted in PBS-T with 5\% BSA; $\beta$-actin (1:10,000), purchased from Abcam (Cambridge, UK) and diluted in PBS-T with 5\% skim milk. Two separate gels were used to detect total and phosphorylated proteins, respectively. Primary antibodies were detected with peroxidase-conjugated secondary antibodies, anti-rabbit IgG (1:2,000; KOMA Biotech, Seoul, Korea) diluted in PBS-T with 5\% skim milk, followed by enhanced chemiluminescence reagents (Amersham Biosciences, Arlington Heights, IL, USA) and exposure to X-ray film. Band intensities were quantified based on densitometric values using Fujifilm Science Lab 97 Image Gauge software (version 2.54) (Fujifilm, Tokyo, Japan).

\section{Statistical analyses}

Statistical analyses were performed using the Sigma Plot version 12.0 (Systat Software, San Jose, CA, USA). The data were analyzed with one-way ANOVA, followed by post-hoc Bonferroni comparisons. Differences between experimental conditions were considered statistically significant when $\mathrm{p}<0.05$.

\section{RESULTS}

\section{Microinjection of LY294002 into the NAcc core decreases ERK phosphorylation levels in this site}

In order to examine our hypothesis that ERM phosphorylation is under the regulation of Akt and GSK3 $\beta$ in the NAcc core, LY294002 was bilaterally microinjected into this site and phosphorylation levels for each molecules were measured. As expected, microinjection of LY294002 into the NAcc core decreased phosphorylation levels of Akt, and subsequently of GSK3 $\beta$, in this site (Fig. 1B, C). The one-way ANOVA conducted on these results revealed significant effects of treatment $\left(\mathrm{F}_{2,15}=57.49, \mathrm{p}<0.001\right.$, for Akt; $F_{2,15}=13.53, p<0.001$, for GSK3 $\beta$, respectively). Further, microinjection of LY294002 into the NAcc core also decreased the phosphorylation levels of ERM in this site (Fig. 1D). The one-way ANOVA conducted on these results showed significant effects between different doses of drugs $\left(\mathrm{F}_{2,15}=25.05, \mathrm{p}<0.001\right)$.

\section{Microinjection of S9 peptide into the NAcc core decreases ERK phosphorylation levels in this site}

In order to confirm whether reduction of GSK3 $\beta$ phosphorylation in the NAcc core actually contributed to the decrease of ERM phosphorylation in this site, we microinjected S9 (see Fig. 2A) bilaterally into the NAcc core with a separate group of rats and measured the phosphorylation levels of GSK3 $\beta$ and ERM. The one-way ANOVA conducted on these results revealed significant effects of treatment $\left(\mathrm{F}_{2,14}=4.59, \mathrm{p}<0.05\right.$, for GSK3 $\beta ; \mathrm{F}_{2,14}=4.39$, $\mathrm{p}$ $<0.05$, for ERM, respectively). Consistent with our previous findings [23], we found that microinjection of S9 into the NAcc core dose-dependently decreased GSK3 $\beta$ phosphorylation in this site ( $p<0.05$ by post-hoc Bonferroni comparisons; higher dose of $S 9$ versus saline) (Fig. 2B). Interestingly, we also found that microinjection of S9 dose-dependently decreased ERM phosphorylation in this site ( $\mathrm{p}<0.05$ by post-hoc Bonferroni comparisons; higher dose of ERM versus saline) (Fig. 2C).

\section{DISCUSSION}

The present results demonstrate that ERM phosphorylation in the NAcc core is decreased by microinjections of S9 peptide, a proposed GSK3 $\beta$ activator, as well as LY294002, PI3 kinase inhibitor, in this site. This is the first direct demonstration, to our knowledge, to indicate that ERM is under the regulation of AktGSK3 $\beta$ signaling in the NAcc core.

There has been no study at all in the literature about what molecular signaling pathways regulate phosphorylation levels of ERM proteins in the NAcc. However, it has been previously shown in our lab that ERM phosphorylation is decreased by acute AMPH in the NAcc core, and interestingly it is recovered back to saline control level when lithium is co-present [12]. Because lithium, among multiple pathways that it affects [24], is known to increase phosphorylation of Akt and GSK3 $\beta[13,14]$, these results plausibly suggest that Akt-GSK3 $\beta$ signaling pathway may position to regulate ERM phosphorylation in the NAcc core. As we hypothesized, we showed in the present study that direct (by reduction of Akt phosphorylation) or indirect (by competition 
with Akt against GSK3 $\beta$ phosphorylation) reduction of GSK3 $\beta$ phosphorylation levels in the NAcc core actually produces the decrease of ERM phosphorylation in this site (see Fig. 3). Although it has been previously shown in neuronal and epidermal cell cultures, and more recently in PC12 cells, that PI3 kinase-Akt signaling pathway, which usually positioned as upstream effectors of GSK3 $\beta$, regulate ERM phosphorylation levels [16,17,25], it is first time to directly show in the NAcc that this pathway also regulates ERM signaling in this region.

It is interesting to compare our present data with a recent report by Jin et al. (2018) [25]. They found that an Akt inhibitor specifically decreased radixin protein levels, but not ezrin and moesin, in PC12 cells. Because we didn't discriminate radixin out of other ERM family proteins, we are not sure whether it would be the case in the NAcc. Nevertheless, it is worth to mention that there is an obvious difference in that they measured protein levels in PC12 cells (in vitro, homogenous), while we did it in the NAcc tissues (in vivo, heterogenous). Interestingly, we previously reported [26] that AMPH rather increases a ratio of phosphorylated to total ERM in this cell, which is opposite of what we observed in the NAcc tissue [12]. Therefore, we think that there might be different regulatory mechanism working depending on the type of cell or brain area that we are looking.

Next, as a down-stream effector of Akt, it has been shown that phosphorylation levels of GSK3 $\beta$ are decreased in the NAcc core by acute cocaine [23]. In parallel, we have found that ERM phosphorylation is also decreased in this site by either acute cocaine [11] or acute AMPH [12]. Put together with these results, the present findings suggest that the effect of psychomotor stimulants on the reduction of ERM phosphorylation in the NAcc core is possibly through a phasic regulation of Akt-GSK3 $\beta$ signaling pathway.

As activation of ERM proteins requires the phosphorylation at the C-terminal region [1,3], the decrease of phosphorylation levels for these proteins in the NAcc core by psychomotor stimulants as shown in our previous reports $[11,12]$ indicates that there might be a chance for a structural change to occur in this region. Supporting this possibility, it was actually shown that dendritic spine density in the NAcc was increased by acute cocaine after 6 $\mathrm{h}$ [27]. Although the detailed mechanism about what molecular pathways are involved in this process is not known, it may be possible that the reduction of the influence of Akt-GSK3 $\beta$ signaling pathway in response to acute psychomotor stimulants may result in decrease of ERM phosphorylation, which then induces a series of cellular events leading to collapse and remodeling of actin cytoskeletons to subsequently produce dendritic spine changes. Even it may be transient, such a change may become a form of metaplasticity [28], and thereby contribute to more endurable structural plasticity in the long run, eventually leading to behavioral changes manifested as addictive behaviors when repeatedly exposed to drugs of abuse [29-31]. These possibilities remain open to be explored in the future.

In conclusion, the present findings showed that ERM phosphorylation in the NAcc core is under the regulation of AktGSK3 $\beta$ signaling pathways, and suggest that they may be positioned as upstream effectors to ERM. As it is not mostly explored yet what ERM proteins function in the brain, especially in the NAcc, our present findings will be a small step toward our understanding for ERM proteins in terms of revealing their signaling pathways in this region. Further, considering their role in structure formation and maintenance, revealing ERM-related signaling pathway in the NAcc, an important region mediating addictive behaviors, as shown in the present findings, has a significant implication to understand structural plasticity and relevant signaling in drug addiction.

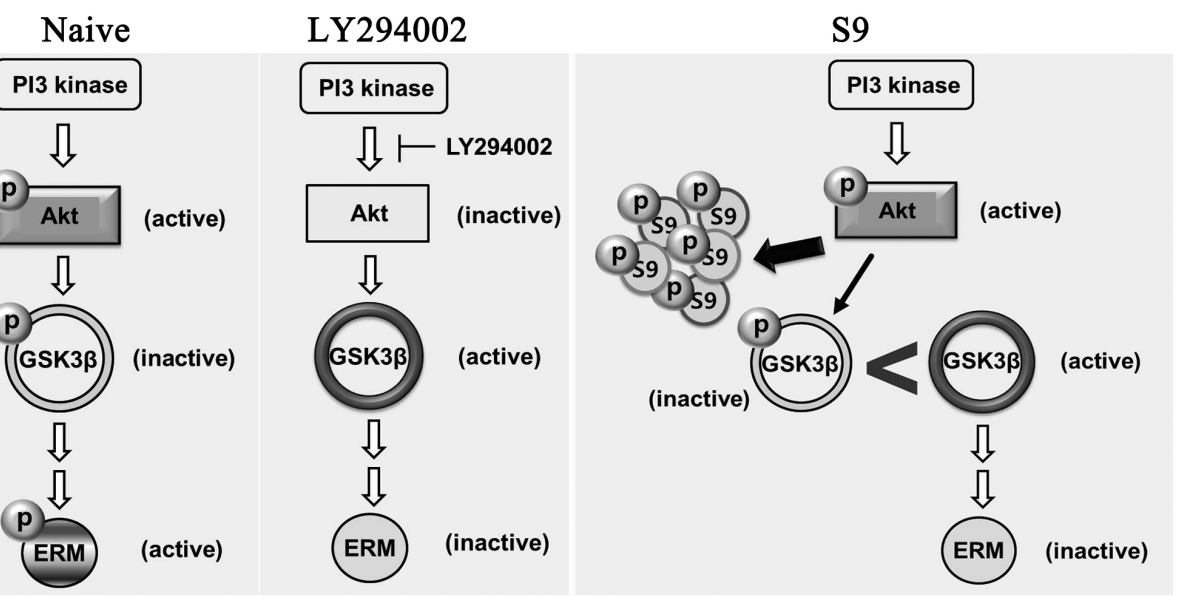

Fig. 3. A hypothetical diagram depicts a signal pathway where activates protein kinase B (Akt) and glycogen synthase kinase 3 3 (GSK3 $\beta$ ) position as possible upstream effectors to ezrin-radixin-moesin (ERM). (Left) GSK3 $\beta$ is normally inactive when phosphorylated, for example, by its upstream effector, Akt, while ERM is active contributing to the maintenance of cell structures. (Middle) When LY294002 is present, as Akt becomes inactive, so does GSK3 $\beta$ active by less chance of phosphorylation. Interestingly, ERM also becomes inactive correspondingly. (Right) With S9 peptide entered, Akt is now competing with S9 against its phosphorylation site of GSK3 3 , so there is more chance that active (un-phosphorylated) form of GSK3 $\beta$ exists. Again with S9, ERM becomes inactive correspondingly, suggesting that it is under the regulation of Akt and GSK3 $\beta$. 


\section{ACKNOWLEDGEMENTS}

This study was supported, by Faculty Research Grant from Yonsei University College of Medicine (6-2013-0148 to JHK), and by the National Research Foundation of Korea (NRF) grants (2018025230 to JHK; 2019R1A2C1011262 to WYK) funded by the Korea government (MSIT).

\section{CONFLICTS OF INTEREST}

The authors declare no conflicts of interest.

\section{REFERENCES}

1. Bretscher A, Edwards K, Fehon RG. ERM proteins and merlin: integrators at the cell cortex. Nat Rev Mol Cell Biol. 2002;3:586-599.

2. Louvet-Vallée S. ERM proteins: from cellular architecture to cell signaling. Biol Cell. 2000;92:305-316.

3. Niggli V, Rossy J. Ezrin/radixin/moesin: versatile controllers of signaling molecules and of the cortical cytoskeleton. Int J Biochem Cell Biol. 2008;40:344-349.

4. Pelaseyed T, Bretscher A. Regulation of actin-based apical structures on epithelial cells. J Cell Sci. 2018;131:jcs221853.

5. Matus A. Growth of dendritic spines: a continuing story. Curr Opin Neurobiol. 2005;15:67-72.

6. Neisch AL, Fehon RG. Ezrin, Radixin and Moesin: key regulators of membrane-cortex interactions and signaling. Curr Opin Cell Biol. 2011;23:377-382.

7. Robbins TW, Cador M, Taylor JR, Everitt BJ. Limbic-striatal interactions in reward-related processes. Neurosci Biobehav Rev. 1989; 13:155-162.

8. Koob GF, Le Moal M. Drug addiction, dysregulation of reward, and allostasis. Neuropsychopharmacology. 2001;24:97-129.

9. Goto Y, Grace AA. Limbic and cortical information processing in the nucleus accumbens. Trends Neurosci. 2008;31:552-558.

10. Nestler EJ. Is there a common molecular pathway for addiction? Nat Neurosci. 2005;8:1445-1449.

11. Kim WY, Shin SR, Kim S, Jeon S, Kim JH. Cocaine regulates ezrinradixin-moesin proteins and RhoA signaling in the nucleus accumbens. Neuroscience. 2009;163:501-505.

12. Kim WY, Jang JK, Shin JK, Kim JH. Amphetamine dephosphorylates ERM proteins in the nucleus accumbens core and lithium attenuates its effects. Neurosci Lett. 2013;552:103-107.

13. Beaulieu JM, Caron MG. Looking at lithium: molecular moods and complex behaviour. Mol Interv. 2008;8:230-241.

14. Beaulieu JM, Del'guidice T, Sotnikova TD, Lemasson M, Gainetdinov RR. Beyond cAMP: The regulation of Akt and GSK3 by dopamine receptors. Front Mol Neurosci. 2011;4:38.

15. Li B, Ren J, Yang L, Li X, Sun G, Xia M. Lithium inhibits GSK3 $\beta$ activity via two different signaling pathways in neurons after spinal cord injury. Neurochem Res. 2018;43:848-856.

16. Gallo G. Semaphorin 3A inhibits ERM protein phosphorylation in growth cone filopodia through inactivation of PI3K. Dev Neurobiol. 2008;68:926-933.

17. Jeon S, Park JK, Bae CD, Park J. NGF-induced moesin phosphorylation is mediated by the PI3K, Racl and Akt and required for neurite formation in PC12 cells. Neurochem Int. 2010;56:810-818.

18. Cencetti F, Bernacchioni C, Bruno M, Squecco R, Idrizaj E, Berbeglia M, Bruni P, Donati C. Sphingosine 1-phosphate-mediated activation of ezrin-radixin-moesin proteins contributes to cytoskeletal remodeling and changes of membrane properties in epithelial otic vesicle progenitors. Biochim Biophys Acta Mol Cell Res. 2019;1866:554-565.

19. Choi JM, Ahn MH, Chae WJ, Jung YG, Park JC, Song HM, Kim YE, Shin JA, Park CS, Park JW, Park TK, Lee JH, Seo BF, Kim KD, Kim ES, Lee DH, Lee SK, Lee SK. Intranasal delivery of the cytoplasmic domain of CTLA-4 using a novel protein transduction domain prevents allergic inflammation. Nat Med. 2006;12:574-579.

20. Dajani R, Fraser E, Roe SM, Young N, Good V, Dale TC, Pearl LH. Crystal structure of glycogen synthase kinase 3 beta: structural basis for phosphate-primed substrate specificity and autoinhibition. Cell. 2001;105:721-732.

21. Frame S, Cohen P, Biondi RM. A common phosphate binding site explains the unique substrate specificity of GSK3 and its inactivation by phosphorylation. Mol Cell. 2001;7:1321-1327.

22. Pellegrino LJ, Pellegrino AS, Cushman AJ. A stereotaxic atlas of the rat brain. New York: Plenum; 1979.

23. Kim WY, Jang JK, Lee JW, Jang H, Kim JH. Decrease of GSK3 $\beta$ phosphorylation in the rat nucleus accumbens core enhances cocaine-induced hyper-locomotor activity. J Neurochem. 2013;125: 642-648.

24. O'Donnell KC, Gould TD. The behavioral actions of lithium in rodent models: leads to develop novel therapeutics. Neurosci Biobehav Rev. 2007;31:932-962.

25. Jin EJ, Ko HR, Hwang I, Kim BS, Choi JY, Park KW, Cho SW, Ahn JY. Akt regulates neurite growth by phosphorylation-dependent inhibition of radixin proteasomal degradation. Sci Rep. 2018;8:2557.

26. Jeong HJ, Kim JH, Jeon S. Amphetamine-induced ERM proteins phosphorylation is through PKC $\beta$ activation in PC12 cells. Korean J Physiol Pharmacol. 2011;15:245-249.

27. Shen HW, Toda S, Moussawi K, Bouknight A, Zahm DS, Kalivas PW. Altered dendritic spine plasticity in cocaine-withdrawn rats. $J$ Neurosci. 2009;29:2876-2884.

28. Abraham WC. Metaplasticity: tuning synapses and networks for plasticity. Nat Rev Neurosci. 2008;9:387-399.

29. Li Y, Acerbo MJ, Robinson TE. The induction of behavioural sensitization is associated with cocaine-induced structural plasticity in the core (but not shell) of the nucleus accumbens. Eur J Neurosci. 2004;20:1647-1654.

30. Robinson TE, Kolb B. Structural plasticity associated with exposure to drugs of abuse. Neuropharmacology. 2004;47 Suppl 1:33-46.

31. Lee KW, Kim Y, Kim AM, Helmin K, Nairn AC, Greengard P. Cocaine-induced dendritic spine formation in D1 and D2 dopamine receptor-containing medium spiny neurons in nucleus accumbens. Proc Natl Acad Sci U S A. 2006;103:3399-3404.

32. Paxinos G, Watson C. The rat brain in stereotaxic coordinates. 5th ed. London: Elsevier Academic; 2004. 


\section{Acknowledgement to Reviewers - 2019}

The Editors of The Korean Journal of Physiology \& Pharmacology (KJPP) would like to thank the following referees who have generously contributed their time and expertise to review manuscripts during the past year. Reviewers' valuable support and contributions have greatly improved the scientific quality of the journal.

\begin{tabular}{|c|c|c|}
\hline Ahn, Jung-Hyuck & Jeon, Songhee & Kim, Young-Hoon \\
\hline Al-Faruque, Hasan & Jeon, Young Jin & Ko, Jae-Hong \\
\hline Bae, Eun Ju & Jeong, Yong Taek & Kong, Eunhee \\
\hline Bae, Jae-Sung & Jeong, Yong Taek & Kook, Hyun \\
\hline Bae, Jong-Sup & Ji, Sang Chun & Kwak, Mi-Kyoung \\
\hline Bae, Soo Hyeon & Jo, Eun-Kyeong & Kwak, Yong Geun \\
\hline Bae, Sunsik & Jun, Jae Yeoul & Kwon, Minsoo \\
\hline Baik, Eun Joo & Jung, Eun Seok & Kwon, Sang-Mo \\
\hline Bang, Hyoweon & Jung, Hanna & Kwon, Tae-Hwan \\
\hline Byun, Kyunghee & Jung, Sung-Cherl & Lee, Bombi \\
\hline Cha, Seok Ho & Kaang, Bong-Kiun & Lee, Chang Hoon \\
\hline Cha, Seung-Kuy & Kang, Dawon & Lee, Choongjae \\
\hline Chae, Hanjung & Kang, Jaeku & Lee, Dae-Heui \\
\hline Chang, Keuna & Kang, Ju-Hee & Lee, Eunee \\
\hline Cho, Jooyoun & Kang, Keon Wook & Lee, Hoi Young \\
\hline Cho, Jungsook & Kang, Youngjin & Lee, HW \\
\hline Cho, Kyung Sook & Ki, Sung Hwan & Lee, Hye-Jeong \\
\hline Cho, Kyung-Ok & Kim, Chi Dae & Lee, Jaehwi \\
\hline Choi, Byung Wook & Kim, Dong-Hoon & Lee, Jin-Koo \\
\hline Choi, Byungmoon & Kim, Dong-Hyun & Lee, Jinu \\
\hline Choi, Hyoungchul & Kim, Dong-Wook & Lee, Jiyoun \\
\hline Choi, Hyun Jin & Kim, Eosu & Lee, Joo Young \\
\hline Choi, Won-Il & Kim, Hwa-Jung & Lee, Jungho \\
\hline Choi, Youn-Hee & Kim, Hyoung Kyu & Lee, Jungho \\
\hline Chun, Sang Woo & Kim, Hyun Jin & Lee, Kyu Pil \\
\hline Chun, Wanjoo & Kim, Hyungbum & Lee, Mi-Ock \\
\hline Chung, Gehoon & Kim, Jae Ho & Lee, Moo Yeol \\
\hline Chung, Jin Woong & Kim, Ja-Eun & Lee, Sang Kook \\
\hline Dai, Jian & Kim, Jee In & Lee, Seogki \\
\hline Ha, Un-Hwan & Kim, Jin Sook & Lee, Seoul \\
\hline Hahm, Ki Baik & Kim, Jong-Hoon & Lee, Seung Jin \\
\hline Han, Ho-Jae & Kim, Joo Young & Lee, Suk-Ho \\
\hline Han, Joo-Hui & Kim, Ka Young & Lee, Sung Hoon \\
\hline Han, Seong Kyu & Kim, Koanhoi & Lee, Yong-Seok \\
\hline Heo, Kyung-Sun & Kim, Kyung Keun & Leem, Chae Hun \\
\hline Huh, Joo Yong & Kim, Min Seok & Lim, Hyeong-Seok \\
\hline Hur, Gang Min & Kim, Myoung-Hwan & Lim, Ji-Hong \\
\hline Hwang, Soon Jung & Kim, Namkug & Lim, Ki Moo \\
\hline Jaggi, Amteshwar & Kim, Sanggeon & Lim, Kyoungmin \\
\hline Jang, Choon-Gon & Kim, Soo Wan & Moon, Seok Jun \\
\hline Jang, Hyun-Jong & Kim, Sun Kwang & Myung, Soon Chul \\
\hline Je, Hyun Dong & Kim, Wonyong & Nam, Hae Jeong \\
\hline Jeon, Byeong Hwa & Kim, Yong Sook & Nam, Joo Hyun \\
\hline
\end{tabular}




Nandakumar, Krishnadas
Oh, Ki-Wan
Oh, Seog Bae
Ortiz, Ortiz
Pan, Zhijian
Park, Byung-Hyun
Park, Hwan-Woo
Park, Jehyun
Park, Jongsun
Park, Joo Min
Park, Kyungsoo
Park, Kyu-Sang
Park, Pil-Hoon
Park, Ryeongyeon
Park, Sangmyun
Park, Seung-Kiel
Park, Song-Kyu
Park, Won Sun
Rhee, Poong-Lyul
Rhyu, Dong Young
Roh, Gu Seob

Ryoo, Sungwoo
Ryu, Ji-Hwan
Safhi, Mohammed
Seo, Joon Beom
Shim, Hyun Soo
Shin, Chan Young
Shin, Eun-Joo
Shin, Hwa-Sup
Shin, Joo-Ho
Shin, Kyung Ho
Singh, Nirmal
So, Insuk
Sohn, Uy Dong
Son, Chang-Gue
Son, Hyeon
Song, Dae-Kyu
Song, Dong-Keun
Song, Wook
Suh, Suk Hyo
Suh, Wonhee
Suh, Yoo-Hun

Suh, YoungHo

Sun, Ming-Jun

Uddin, Md Jamal

Um, Jar-Young

Um, Sung Hee

Wang, Li

Woo, Hyun Ae

Wu, Jianhua

Wu, Xianjin

Yang, Chae Ha

Ye, Sangkyu

Yi, Young-Su

Yim, Hyungshin

Yoon, Jihoon

You, Ho-Jin

Youm, Jae Boum

Youn, Dong

Yu, Zengli

Yunjo, Soh

Zendehdel, Morteza

Zhang, Qi 